\title{
ASPECTS NUTRITIONNELS
}

\section{L'utilisation des protéines du lait à des fins diététiques et thérapeutiques en alimentation humaine}

\author{
par \\ V. WENNER*
}

\section{Ré $\mathbf{s}$ u $\mathrm{m}$ é}

Cette présentation est un résumé critique des connaissances actuelles de la valeur diététique et thérapeutique des protéines du lait en alimentation humaine. Elle est un complément à celle faite par le même auteur à Paris en 1978 sur les effets diététiques du lait de vache et de ses composés.

Les technologies utilisées dans l'industrie pour séparer, enrichir et purifier les protéines tels que l'ultrafiltration (concentration), la filtration sur gel (purification par exclusion) et la séparation par échangeur d'ions (extraction, purification) permettent la production de protéines pures à une échelle suffisante pour entreprendre des essais valables en diététique et en thérapeutie.

Actuellement, peu de résultats valables peuvent être mentionnés. Ceci s'explique par le fait que dans le domaine de la diététique et des thérapies, les essais sur animaux ne sont pas valables et il est impossible de les extrapoler et les adapter à l'homme. Pour obtenir un effet diététique, il faut définir une déficience métabolique à traiter par les protéines du lait. Pour avoir un effet thérapeutique, une maladie ou une lésion doit être guérissable par les protéines lac-

* Département Recherche, Société d'Assistance Technique pour Produits Nestlé S.A., Case postale 88, CH-1814.

La Tour-de-Peilz (Suisse). 
tiques. Présentement seul le nourrisson se prête à des essais pour obtenir sans trop de difficultés des résultats exploitables.

Pour le moment on peut dire que seules les protéines présentes dans le lait en faibles quantités manifestent des activités biologiques intéressantes (immunoglobulines, $\alpha$-lactalbumine, lactoferrine, lysozymes, etc.). Les immunoglobulines exclusivement ont fait preuve d'une activité thérapeutique clairement définissable.

En conclusion, on peut prétendre que les protéines du lait et leurs hydrolysats enzymatiques sont des substances valables, biologiquement actives, mais que nous ne disposons pas encore d'hypothèses de travail suffisamment bonnes pour lancer des études à long terme sur des êtres humains.

\section{S u m m a ry}

A critical overview is given on the effective or presumable benefits of cow's milk proteins in human nutrition. This presentation is to be considered as a complement to the conference given at Paris in 1978 by the same author. In 1978, an overview was given on the dietetic properties of cow's milk and different milk constituents.

The presently available and industrially applicable technologies for protein enrichment, and/or purification such as ultrafiltration (concentration), gelfiltration (purification by exclusion) and ion exchange (purification by selective extraction) make it possible to separate the major milk protein fractions in amounts large enough to allow the study of their behaviour as dietetic and therapeutic agents.

For the time being, scientific and valuable data are few and precise definitions are often unavailable. Definitions are lacking to know what kind of metabolic deficiencies could be improved by feeding milk proteins (dietetic effects) or what illnesses or lesions are cured by milk proteins (therapeutical effects).

This situation is explained by the difficulty to carry out valuable experimental work in the nutritional, dietetical and therapeutical fields on animal models. Such experiments are difficult to execute on human beings with the exception of newborn or small infants, who are, of course, not comparable with adults and even less with elderly people.

The present paper gives certain results confirmed in clinical trials on the therapeutical effects of immunoglobulins. Other effects of milk proteins are to be defined as the action of specifically active groups of proteins (iron binding of lactoferrine, tryptophane concentration in $\alpha$-lactalbumin, -SER-PO, groups in casein, a.s.f.) or as synergistic effects with other milk constituents. Other observations can be interpreted as stimulation of cell growth by peptides (protein 
hydrolysate) or as a stimulation of the gastric or pancreatic secretion, a.s.f.

It is felt that the milk proteins are valuable biologically active substances, especially the minor protein fractions of milk, but we lack perhaps good working hypothesis and we certainly encounter enormous difficulties to achieve long term studies on human beings.

\section{INTRODUCTION}

Depuis 20 à 30 ans, on s'intéresse à des études qui ont pour but de mieux définir les effets diététiques et thérapeutiques des protéines du lait. Les nombreux cas de malnutrition et de famine dans certains pays d'Europe après la deuxième guerre mondiale ont suscité l'étude du rétablissement de millions de personnes à l'aide de protéines et d'hydrolysats de protéines dérivés du lait. A cette époque-là, les laits concentrés sucrés, ou non sucrés stérilisés et les laits en poudre étaient utilisés comme apport diététique en nutrition humaine. Les hydrolysats de caséine ont été utilisés comme éléments thérapeutiques en cas de malnutrition grave où les fonctions digestives étaient déjà gravement compromises.

Une orientation scientifique et précise des études concernant la valeur diététique des protéines lactiques est devenue possible il y a seulement une dizaine d'années et ceci pour trois raisons principales :

- Manque d'hypothèses de travail précises et acceptées ou acceptables.

- Manque de technologies valables pour isoler les protéines du lait, en particulier celles ayant une activité biologique connue (immunoglobuline, lactoferrine, etc.). L'ultrafiltration (concentration des protéines), la filtration sur gel (exclusion des protéines de leur milieu) et l'extraction sélective par exemple de la $\beta$-lactoglobuline par échangeur d'ions (Sphérosil, Rhône-Poulenc) ont apporté des solutions industrielles à ce problème (voir également tableau 1).

- Manque d'une exploitation scientifique et rationnelle du potentiel de la vache laitière pour la biosynthèse dirigée ou dirigeable de certaines protéines (voir immunoglobulines spécifiques ci-après). Le tableau 2 donne une idée de l'énorme progrès réalisé pour augmenter le rendement de la vache laitière. Une prochaine étape de la sélection sera sans doute l'étude des possibilités de modification de la composition du lait afin de mieux l'adapter à certains besoins de l'homme.

Il nous paraît intéressant (voir tableau 3) de situer les protéines du lait par rapport aux principales protéines alimentaires produites à l'échelle mondiale. 450 millions de tonnes de lait sont équivalentes à 15 millions de tonnes de protéines ou 12 à $15 \%$ du tonnage des 


\section{TABLEAU 1}

Production mondiale des protéines lactiques sous forme de lait, fromages et lactosérum

(voir également Hambreus, 1978)

\begin{tabular}{l|c|c}
\hline & $\begin{array}{c}\text { Du lait total } \\
\text { (millions } \mathrm{t})\end{array}$ & $\begin{array}{c}\text { Dans le lactosérum } \\
\text { des fromageries } \\
(\mathrm{t})\end{array}$ \\
\hline Caséines & 12 & - \\
$\beta$-lactoglobulines & 1,3 & 200000 \\
$\alpha$-lactalbumines & 0,5 & 70000 \\
Sérum albumine & 0,1 & 15000 \\
Immunoglobulines & $0,3^{*}$ & 45000 \\
Lactoferrine & $<0,02$ & $<3000$ \\
Lysozyme & $<0,01$ & $<2000$ \\
Matières azotées & 0,8 & 120000 \\
non protéiques $(\mathrm{N} \times 6,4)$ & & \\
\hline
\end{tabular}

* On peut ajouter 0,1 à $0,15-10^{6} \mathrm{t}$ d'immunoglobulines du lait colostral et du lait de fin de lactation considéré comme impropre à la consommation.

TABLEAU 2

Productivité de la vache laitière dans les pays industrialisés

\begin{tabular}{l|l|c}
\hline & & Lait $\mathrm{kg} / \mathrm{an}$ \\
\cline { 2 - 3 } En 1920 & $\begin{array}{l}\text { moyenne en Suisse selon les races } \\
\text { En } 1960\end{array}$ & 2500 à 3000 \\
& moyenne en Suisse selon les races & 3000 à 3500 \\
& fermes modèles (Suisse) & 4500 \\
En 1980 & fermes de sélection (Danemark) & 8000 \\
& fermes modèles (U.S.A.) & 8000 \\
& vache record (U.S.A.) & 25000 \\
\hline
\end{tabular}


protéines des céréales. Toutefois, si l'on tient compte de la valeur nutritionnelle et biologique des protéines du lait, on peut estimer qu'elle représente environ $20 \%$ de celle des céréales. Ces valeurs sont basées sur une productivité de la vache laitière (pour la moyenne mondiale) d'un maximum de $3000 \mathrm{~kg} / \mathrm{an}$. Toutefois, le potentiel moyen de la vache laitière (voir tableau 2) peut certainement être estimé à $5000 \mathrm{~kg}$ ou plus.

Les protéines du lait pouvant être utilisées à des fins diététiques et thérapeutiques on peut se demander comment exploiter ce potentiel de façon économique et selon quels principes industriels et rationnels?

\section{VALEUR NUTRITIONNELLE DES PROTEINES DU LAIT}

La valeur nutritionnelle des protéines du lait est si élevée que l'on utilise ces protéines en tant que protéines de référence dans les essais sur animaux.

La valeur nutritionnelle dépend de la composition en acides aminés et de la digestibilité de ces protéines. C'est avant tout la composition équilibrée en acides aminés essentiels qui doit être considérée pour juger de la valeur nutritionnelle d'une protéine. Le complexe total des matières azotées du lait de vache (et des laits de tous les mammifères selon le besoin des espèces) est parfaitement bien équilibré mais, comme le montre le tableau 4, les différentes protéines prises individuellement ne le sont pas. Il y a là des considérations intéressantes à discuter si nous voulons analyser en détail la valeur diététique en plus de la valeur nutritionnelle de ces protéines.

La valeur nutritionnelle des protéines du lait est discutée plus en détail par P. Vert (digestion des protéines et développement du nourrisson), E. Renner (amélioration des aliments végétaux par les protéines du lactosérum) et J.P. Rampal (utilisation des protéines du lait comme milieu nutritif dans les cultures cellulaires) dans les exposés qui suivront.

\section{VALEUR DIETETIQUE ET/OU THERAPEUTIQUE DES PROTEINES DU LAIT}

Il est difficile de donner une définition précise et scientifiquement valable des effets diététiques ou thérapeutiques préconisés pour les protéines lactiques en alimentation humaine. Les raisons de ces difficultés sont multiples et nous en rappelons quelques-unes:

a) Pour définir un effet diététique, il faut pouvoir déterminer une déficience métabolique de l'homme qui peut être améliorée par une 


\section{TABLEAU 3}

Production annuelle mondiale des principales protéines alimentaires

\begin{tabular}{l|c|c}
\hline & $\begin{array}{c}\text { Matières premières } \\
\text { (millions } \mathrm{t})\end{array}$ & Protéines (millions t) \\
\hline Céréales & 1500 & 100 à 120 \\
Viandes et poissons & 150 à 200 & $\sim 40$ \\
Soja & 100 & $\sim 40$ \\
Lait de vache & 450 & $\sim 15$ \\
\hline
\end{tabular}

\section{TABLEAU 4}

Déficience ou surabondance d'acides aminés des protéines du lait de vache prises en considération individuellement

\begin{tabular}{l|c|c}
\hline & Déficience en & Riche en \\
\hline$\beta$-lactoglobulines & $\begin{array}{c}\text { Cystine } \\
\text { (Tryptophane) }\end{array}$ & $\begin{array}{c}\text { Proline } \\
- \text { Ser-PO }\end{array}$ \\
$\alpha$-lactalbumine & - & $\begin{array}{c}\text { Leucine } \\
\text { Lysine } \\
\text { (Tryptophane) } \\
\text { Tryptophane } \\
\text { Lysine } \\
\text { Cystine }\end{array}$ \\
\hline
\end{tabular}

alimentation contenant des protéines du lait ou une protéine spécifique du lait. Il est difficile et souvent impossible de déterminer un modèle d'expérimentation valable sur l'animal.

b) Pour définir un effet thérapeutique, il faut connaître une maladie curable ou des lésions réparables par une alimentation contenant des protéines du lait. Le modèle animal est encore plus difficile à trouver et il ne reste que l'essai sur l'homme en clinique pour donner des preuves de l'efficacité d'un traitement.

c) Il y a dans la pensée traditionnelle de toutes les populations toutes sortes d'interprétations, souvent mystiques, sur les bienfaits du lait. Ces croyances peuvent avoir un fond de vérité mais dans la 
plupart des cas elles ne sont pas vérifiables par des expériences scientifiques.

d) Peut-être y a-t-il dans la pensée traditionnelle des hommes de science aussi des soi-disant «acquis» qu'il faudrait mettre en doute de temps à autre (voir Descartes) afin d'éviter de nous barrer la route qui pourrait nous mener à la découverte de nouvelles vérités?

Pour mieux illustrer ces questions et constatations, nous représentons dans la figure 1 un ancien essai sur rats qui a eu pour but de vérifier la tolérance de trois formules de lait:

- poudre de lait de vache $30 \%$ de protéines $(80 \%$ de caséine, $20 \%$ de lactalbumines), $28 \%$ de matière grasse ;

- poudre de lait de vache $10 \%$ de protéines (40\% de caséine, $60 \%$ de lactalbumines), $27 \%$ de matière grasse ;

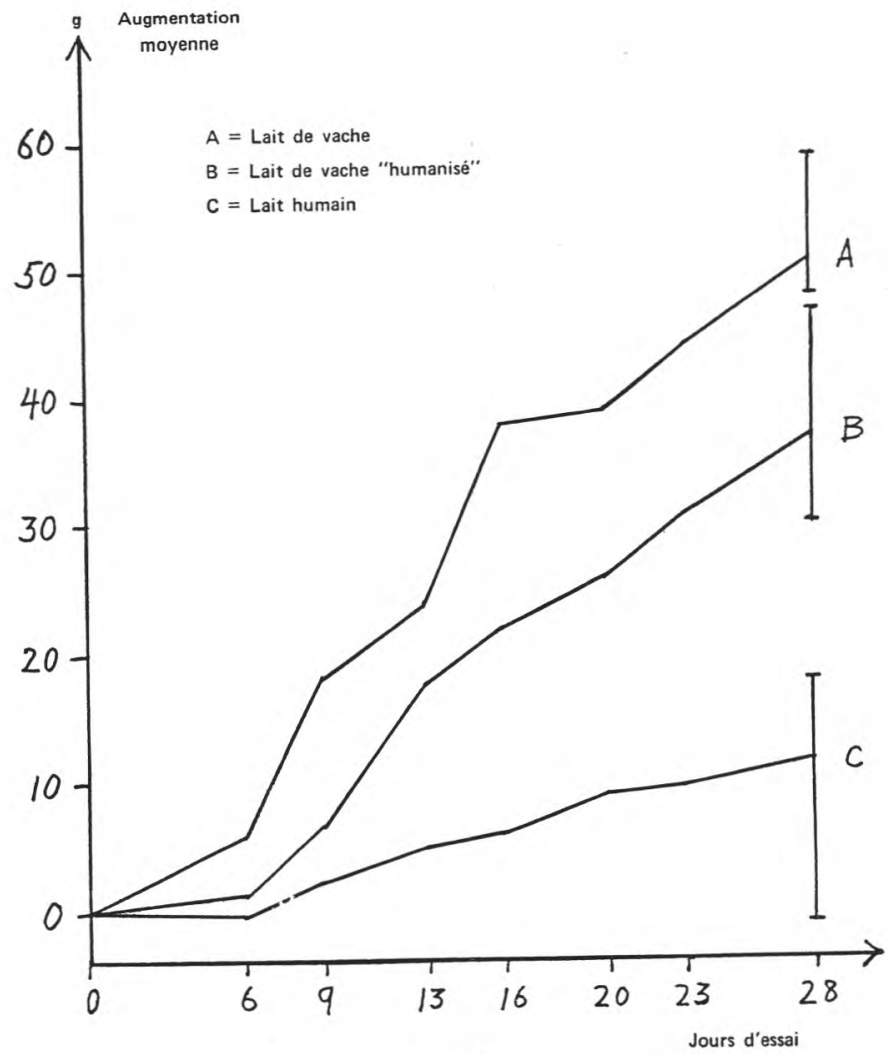

fig. 1 
- lait humain séché $10 \%$ de protéines (35\% de caséine, $65 \%$ de lactalbumines), $30 \%$ de matière grasse.

Le résultat est étonnant. Le lait humain de valeur nutritionnelle égale au lait de vache humanisé n'est pas toléré. Comme on pouvait s'y attendre du fait de sa teneur insuffisante en protéines, le lait humanisé donne une croissance moins rapide que le lait de vache. Les rats nourris au lait humain souffrent de troubles digestifs graves, mangent la totalité de leurs excréments, ont un caecum énorme et l'intestin inflammé, signes probables d'une allergie au lait humain. La flore intestinale est composée de lactobabilles (Bifidum) et de coliformes normaux, donc pas de diarrhée infectieuse.

Cet exemple devrait nous rendre attentif au fait que seule l'expérimentation sur l'homme nous autorise à donner des avis sur la valeur diététique ou thérapeutique du lait ou de l'un ou l'autre de ses constituants.

A ce jour, le seul organisme valable pour étudier la valeur diététique des protéines du lait est le nourrisson. Il est facile de disposer d'un certain nombre de sujets pouvant être contrôlés dans les maternités, en clinique, en institution (pouponnière, orphelinat) et même dans leur environnement normal qui est la famille. Des résultats consolidés, statistiquement valables et exploitables, peuvent être produits avec l'effort voulu et la patience nécessaire.

Actuellement très peu de résultats sont disponibles sur les effets diététiques et thérapeutiques des protéines du lait et il est difficile de les interpréter.

Dans le tableau 5 nous avons réunis des résultats considérés comme sûrs et des solutions logiques, même si les preuves scientifiques font défaut. Les remarques suivantes s'imposent:

\section{Immunoglobulines}

On peut immuniser des vaches laitières contre des bactéries pathogènes pour l'homme ou contre des toxines. Les immunoglobulines extraites du lait de ces vaches peuvent être incorporées dans une formule de lait pour nourrissons afin de combattre des infections intestinales comme publié par Hilpert et al. (1975), Mietens et al. (1979), Pahud et al. (1981). Conformément aux résultats présentés déjà en 1892 par Ehrlich et aux essais faits par Petersen et Campel publiés en 1955, les anticorps du lait (immunoglobulines ou leurs fractions actives) peuvent aussi traverser la paroi intestinale et agir sur l'organisme. Il a été ainsi possible de guérir $100 \%$ de jeunes rats ayant reçu par injection sous-cutanée, selon le test classique, une dose mortelle de tetanus toxine (Hilpert, communication privée).

\section{Lactoferrine}

Il devrait paraître logique que la lactoferrine chargée de $\mathrm{Fe}$ puisse combattre des anémies. La question est de savoir si cette 


\section{TABLEAU 5}

Utilisation des protéines du lait pour obtenir des effets diététiques ou thérapeutiques

\begin{tabular}{|c|c|}
\hline Etat maladif & $\begin{array}{l}\text { Protéines actives ou } \\
\text { à conseiller et références }\end{array}$ \\
\hline Infections intestinales & $\begin{array}{l}\text { Immunoglobulines }[7,11,17,19,20] \\
\text { (Lysozyme [21]) } \\
\text { (Lactoferrine [2, 10, 21]) }\end{array}$ \\
\hline Intoxications & $\begin{array}{l}\text { Immunoglobulines } \\
\text { [HILPERT, communication privée] }\end{array}$ \\
\hline Anémies (carence $\mathrm{Fe}$ ) & Lactoferrine ? $[2,10,21]$ \\
\hline Cystinose (déficience métabolique) & $\begin{array}{l}\alpha \text { et } \beta \text {-caséines } \\
\text { (év. caséine complète) }\end{array}$ \\
\hline $\begin{array}{l}\text { Sécrétion gastrique déficiente } \\
\mathrm{HCl}, \text { Pepsine) }\end{array}$ & Protéines du lactosérum \\
\hline Sécrétion pancréatique déficiente & $\begin{array}{l}\text { Hydrolysats enzymatiques des } \\
\text { protéines du lait }\end{array}$ \\
\hline $\begin{array}{l}\text { Malnutrition (réhabilitation, } \\
\text { traitement post-opéraotire) }\end{array}$ & $\begin{array}{l}\text { Protéines du lactosérum ou } \\
\text { hydrolysats de protéines lactiques }\end{array}$ \\
\hline $\begin{array}{l}\text { Insuffisance du métabolisme } \\
\text { des acides nucléiques } \\
\text { (protéines cellulaires) }\end{array}$ & Protéines lactiques \\
\hline $\begin{array}{l}\text { Régénération de la peau } \\
\text { (par analogie régénération } \\
\text { de la muqueuse intestinale) }\end{array}$ & $\begin{array}{l}\text { Protéines du lactosérum ou } \\
\text { hydrolysats de protéines lactiques } \\
{[12,22]}\end{array}$ \\
\hline $\begin{array}{l}\text { Psychobiologie : } \\
\text { état nerveux central déficient } \\
\text { (sommeil, appétit, humeur, etc.) }\end{array}$ & $\begin{array}{l}\alpha \text {-lactalbumine } \\
\text { (Tryptophane }[5,8,18,23,25])\end{array}$ \\
\hline $\begin{array}{l}\text { Allergies aux protéines lactiques } \\
\text { (ou autres) }[6,9,14,16]\end{array}$ & Hydrolysats des protéines lactiques \\
\hline
\end{tabular}

protéine transporterait elle-même, ou en tant que peptide, le Fe à travers la paroi intestinale (voir également Ribadeau-Dumas, 1978, et Hambreus, 1978). Actuellement l'isolation ou l'enrichissement de la lactoferrine du lait de vache n'est pas encore possible pour faire des tests in vivo dans le traitement des anémies étant donné sa faible concentration dans le lait (voir tableau 1).

Présentement, le rôle de la lactoferrine en alimentation est plutôt défini comme agent séquestrant du Fe libre des aliments privant 
ainsi les bactéries pathogènes intestinales du fer dont elles ont un besoin essentiel pour leur croissance. On obtiendra alors un effet bactériostatique grâce à la lactoterrine (voir aussi Braun, 1976, et Ribadeau-Dumas, 1978).

\section{Caséines}

Le complexe caséinique du lait est pauvre en cystine $(0,2$ à $0,3 \%)$ tandis que les $\alpha$ - et $\beta$-caséines sont exemptes de cystine. Dans les cas assez rares d'intolérance à cet acide aminé (cystinose), la caséine complète ou libérée de sa teneur en k-caséine sera la protéine choisie pour alimenter en protéines nécessaires les malades atteints de cystinose.

La caséine du lait de vache est très riche en sérine-phosphate. Les hypothèses suivantes peuvent être émises :

- La caséine et ses peptides peuvent intervenir dans le transport (résorption intestinale) de $\mathrm{Ca}, \mathrm{Mg}, \mathrm{Fe}$ et oligoéléments.

- $\mathrm{La}-\mathrm{Ser}-\mathrm{PO}_{4}$ pourrait intervenir dans l'organisme en croissance pour accélérer ou améliorer l'ossification du squelette. Des corrélations existent entre la composition en protéines et en calcium des laits de différentes espèces (voir Aebi, 1965). Il pourrait être intéressant d'approfondir l'étude des corrélations entre les teneurs caséine, en - Ser- $\mathrm{PO}_{4}$ du lait et la vitesse de l'ossification.

- La $-\mathrm{Ser}-\mathrm{PO}_{4}$ pourrait avoir le rôle de donateur de $-\mathrm{PO}_{4}$ estérifié, nécessaire à tous les tissus de l'organisme (analogie méthionine comme donateur de groupes méthyle).

\section{Protéines du lactosérum}

De façon générale on peut prétendre que dans tous les cas d'insuffisance des fonctions digestives (malnutrition grave, déficience de la sécrétion gastrique ou pancréatique, intervention chirurgicale, etc.), l'application des protéines du lactosérum ou de leurs hydrolysats enzymatiques est à conseiller. C'est la facilité et la rapidité de leur hydrolyse in vitro et in vivo et leur richesse en acides aminés essentiels bien équilibrés qui font d'elles les protéines de choix pour un rétablissement efficace.

Dans ce contexte, il faut souligner (voir tableau 6) l'effet dermatologique des protéines du lactosérum et de leurs hydrolysats. En effet, depuis plus de 40 ans, on trouve des préparations sur le marché qui axent leur publicité sur la régénération de la peau. On a pu prouver que c'étaient surtout les hydrolysats qui accéléraient la cicatrisation des plaies (1967). Toutefois, ce phénomène n'est toujours pas expliqué. Il pourrait s'agir d'une "nourriture " de la peau au niveau cellulaire (voir Klagsbrun (1978), milieux nutritifs de cultures de cellules in vitro), d'un agent qui contiendrait des facteurs stimulant la croissance (voir Iik-Nan Chou, 1979) ou d'un 
TABLEAU 6

Préparations de protéines lactiques proposées pour leurs effets cosmétiques, dermatologiques et/ou " actifs"

\begin{tabular}{l|l}
\hline \multicolumn{1}{c|}{ Produit/Pays } & \multicolumn{1}{c}{ Applications proposées } \\
\cline { 2 - 2 } $\begin{array}{l}\text { Fissan/Allemagne } \\
\text { (lactalbumine ou hydrolysat) }\end{array}$ & $\begin{array}{l}\text { - peau fragile } \\
\text { Nutrilans/Allemagne } \\
\text { (hydrolysats) }\end{array}$ \\
$\begin{array}{l}\text { Suc-Facial/France } \\
\text { (acides aminés ou hydrolysat) }\end{array}$ & - cosmétique du cuir chevelu \\
$\begin{array}{l}\text { Hydrolysat de lactalbumine/Suisse } \\
\text { (Brevet [22]) }\end{array}$ & - activateur dermatologique \\
$\begin{array}{l}\text { Lacatmine/France } \\
\text { (protéines solubles du lait) }\end{array}$ & - principes azotés actifs \\
$\begin{array}{l}\text { Edamine/U.S.A. } \\
\text { (hydrolysat de lactalbumine) }\end{array}$ & - apport nutritionnel \\
$\begin{array}{l}\text { N-Z Amine/U.S.A. } \\
\text { (hydrolysat de caséine) }\end{array}$ & - protéine soluble \\
\hline
\end{tabular}

phénomène lié à l'humidification de la peau par l'hygroscopicité de ces hydrolysats.

Si l'on défini ces effets bénéfiques au niveau de la peau comme étant un stimulateur de croissance (régénération), on pourait espérer un résultat identique en ce qui concerne la muqueuse intestinale. Dans ce cas, les protéines du lactosérum devraient avoir un effet diététique et thérapeutique à la suite de lésions de cette muqueuse provoquées par des infections, des ulcères ou par une intervention chirurgicale.

\section{Alpha-lactalbumine}

Comme la $\beta$-lactoglobine (voir référence publiée par Braunitzer, $1973)$, tout en étant plus riche en tryptophane $(5,2 \%)$ et en cystine $(5,5 \%)$ (voir séquence publiée par Brew, 1970), l'a-lactalbumine a une composition très exceptionnelle. Malheureusement cette protéine est difficile à extraire à l'échelle industrielle sous une forme enrichie ou pure. La matière première de choix est le lactosérum de fromagerie (voir tableau 1).

Les recherches faites par Brezinova (1972), Fernstrom (1974), Moller (1980), Southwell (1972) et Wurtman (1982) sur l'influence 
des acides aminés aromatiques au niveau cérébral ont permis de penser que le tryptophane libre dans une diète riche en hydrates de carbone aurait des effets sédatifs. On peut alors se demander si la $\beta$-lactoglobuline et surtout l' $\alpha$-lactalbumine pourraient avoir un effet sédatif, étant donné que l'hydrolyse de ces protéines par les protéases pancréatiques est extrêmement rapide. Si l'on se réfère une fois de plus au nourrisson alimenté uniquement avec un lait riche en lactalbumine (lait "humanisé "), on atteint une dose de 5 à $6 \mathrm{mg}$ de tryptophane par $\mathrm{kg}$ du poids corporel. Une dose de $10 \mathrm{mg}$ a été reconnue comme étant suffisante (voir Fernstrom, 1974) pour avoir un effet sédatif au niveau cérébral. D'autres effets (par exemple modification de l'appétit) ont été signalés par R. Wurtman (1982). Ces derniers ne devraient pas être classés comme effets diététiques mais plutôt psychologiques, influençant le comportement de l'homme. En ce qui concerne le sommeil il s'agirait d'un régulateur, apportant le sentiment si recherché de "bien-être".

\section{Les protéines du lait remplaçant d'autres protéines animales}

Les protéines du lait remplacent avantageusement d'autres protéines animales (cellulaires) comme la viande, le poisson, etc., car elles se laissent facilement isoler sous une forme très pure, exempte d'acides nucléiques. Ces acides nucléiques sont souvent difficilement métabolisables (personnes âgées, déficiences métaboliques) et il vaut mieux les éliminer des diètes. Les protéines du lait, ayant une composition équilibrée en acides aminés et étant facilement digérées, sont préférables aux protéines végétales (légumineuses), pauvres en acides nucléiques et en général moins bien équilibrées et moins digestes (voir également teneur en facteurs antitrypsine).

\section{Hydrolysats de protéines et allergies}

Par définition, les allergies sont provoquées par des macromolécules se manifestant comme antigènes incitant ainsi l'organisme à fabriquer des anticorps spécifiques. Selon les récentes publications de Bürgin-Wolff et al. (1980), on peut prétendre que, dans des individus non-allergiques, toutes les protéines alimentaires produisent des anticorps détectables dans $80 \%$ des cas. McLaughlan et al. (1981) confirment assez bien ces résultats. Les publications de Halpern et al. (1973) et de Kjellman et Johanson (1979) démontrent clairement qu'il y a peu d'individus qui développent des allergies seulement aux protéines du lait de vache ou à la $\beta$-lactoglobuline (qui n'est pas contenue dans le lait humain). Ces allergies sont plutôt provoquées par:

- soit une persorption de protéines par l'intestin pendant les premières semaines de la vie du nouveau-né ;

- soit un fonctionnement déficient ou exagéré du système d'immuno-défense ; 
- soit une surcharge en protéines non tolérable administrée au nourrisson (trop de nutriments, fonction digestive déficiente) ;

- soit un maltraitement thermique ou mécanique exagéré par les technologies appliquées (voir tableau 7 ci-après) altérant les protéines ou le système qui les entoure.

Il est tout à fait possible de produire des hydrolysats enzymatiques de protéines ultrafiltrés qui ne contiennent plus de macromolécules et qui, dans l'essai antigène-anticorps in vitro, ne donnent plus de réactions positives. En nutrition les hydrolysats enzymatiques ont l'avantage de garder intacts les acides aminés essentiels des protéines lactiques et de ne pas augmenter de façon notable la pression osmatique dans les produits reconstitués.

\section{EFFETS DIETETIQUES DES PROTEINES LACTIQUES DUS A DES SYNERGISMES AVEC D'AUTRES CONSTITUANTS DU LAIT}

Contrairement aux expérimentations pharmacologiques, l'expérimentation en alimentation entreprise pour déceler des effets diététiques doit tenir compte de tous les composés du régime donné. Il est donc possible que l'effet diététique d'une substance soit renforcé (synergisme) ou neutralisé par une autre. Ceci pourrait expliquer les "croyances", "mythes" ou opinions de certains consommateurs.

Un exemple type est le yaourt ou le fromage. Dans ces deux cas, la fermentation microbienne a un effet diététique, sorte de prédigestion du lait ou de la caséine, ressenti comme bénéfique. Les constituants protéiques du lait restent les mêmes.

Un autre exemple négatif ou "anti-diététique " est l'intolérance au lactose par manque de lactase intestinale. Dans ce cas il faut éliminer le lactose pour faire bénéficier l'individu de l'effet diététique de la protéine.

On peut également proposer des études à entreprendre pour mieux comprendre les synergismes où les protéines devraient améliorer l'absorption et l'utilisation des oligoéléments (lactoferrine? caséine?).

Un autre effet diététique des protéines du lactosérum peut être examiné pour favoriser l'implantation d'une flore intestinale du type B./Bifidum ou L. Bifidus. Cet effet apparaît si le lait a un faible pouvoir tampon (peu de phosphates), s'il a un pouvoir réducteur élevé (lactose, vitamine $\mathrm{C}$, teneur en cystine de la lactalbumine, etc.), si sa teneur en lactalbumine, ou celle de son hydrolysat, est élevée. Lorsque la flore B./Bifidum s'installe, le $\mathrm{pH}$ des 
selles tombe à $\mathrm{pH}<6$ ce qui entraîne une bonne solubilisation des sels calciques et magnésiques (phosphates, stéarate, citrates devenant peu solubles à $\mathrm{pH}>6$ ), d'où une meilleure utilisation possible de ces sels.

On peut encore mentionner un autre effet des protéines du lait et du lactosérum. Ce dernier est basé sur leur rapide digestibilité avec une libération d'acides aminés et de peptides qui apparaissent immédiatement dans le sang stimulant probablement la sécrétion gastrique et pancréatique.

Finalement nous mentionnons l'effet émulgateur des peptides des protéines lactiques (voir Jost, 1982) qui peuvent améliorer la digestibilité des matières grasses.

Tous ces synergismes devraient attirer notre attention sur les effets des protéines du lait qui pourraient être utilisés pour prévenir des carences et des dérangements métaboliques.

\section{TRAITEMENTS TECHNOLOGIQUES ET QUALITE DIETETIQUE ET/OU THERAPEUTIQUE DES PROTEINES}

Quelques traitements technologiques sont énumérés dans le tableau 7. Il faut les prendre en considération si l'on veut évaluer correctement la valeur biologique, nutritionnelle, diététique et thérapeutique des protéines ou des hydrolysats de protéines. C'est tout au long du chemin, de la ferme au consommateur, que les traitements technologiques du lait doivent être contrôlés si l'on veut préserver les effets diététiques et thérapeutiques.

\section{CONCLUSIONS}

Les connaissances accumulées à ce jour permettent de définir quelques effets diététiques et thérapeutiques des protéines du lait en alimentation humaine.

Il nous semble que ces protéines devront faire l'objet d'études approfondies et ceci dans un futur immédiat. Ces études sont pluridisciplinaires et vont de la sélection de vaches laitières aux essais en clinique sur l'homme.

Le lait, en tant que matière première alimentaire pour l'homme, contribue de façon essentielle aux besoins actuels et futurs en protéines. Il est disponible en grande quantité mais reste encore mal utilisé, spécialement si l'on se réfère au lactosérum de fromagerie. 


\section{TABLEAU 7}

Traitements technologiques détériorant la valeur biologique du lait et des protéines du lait

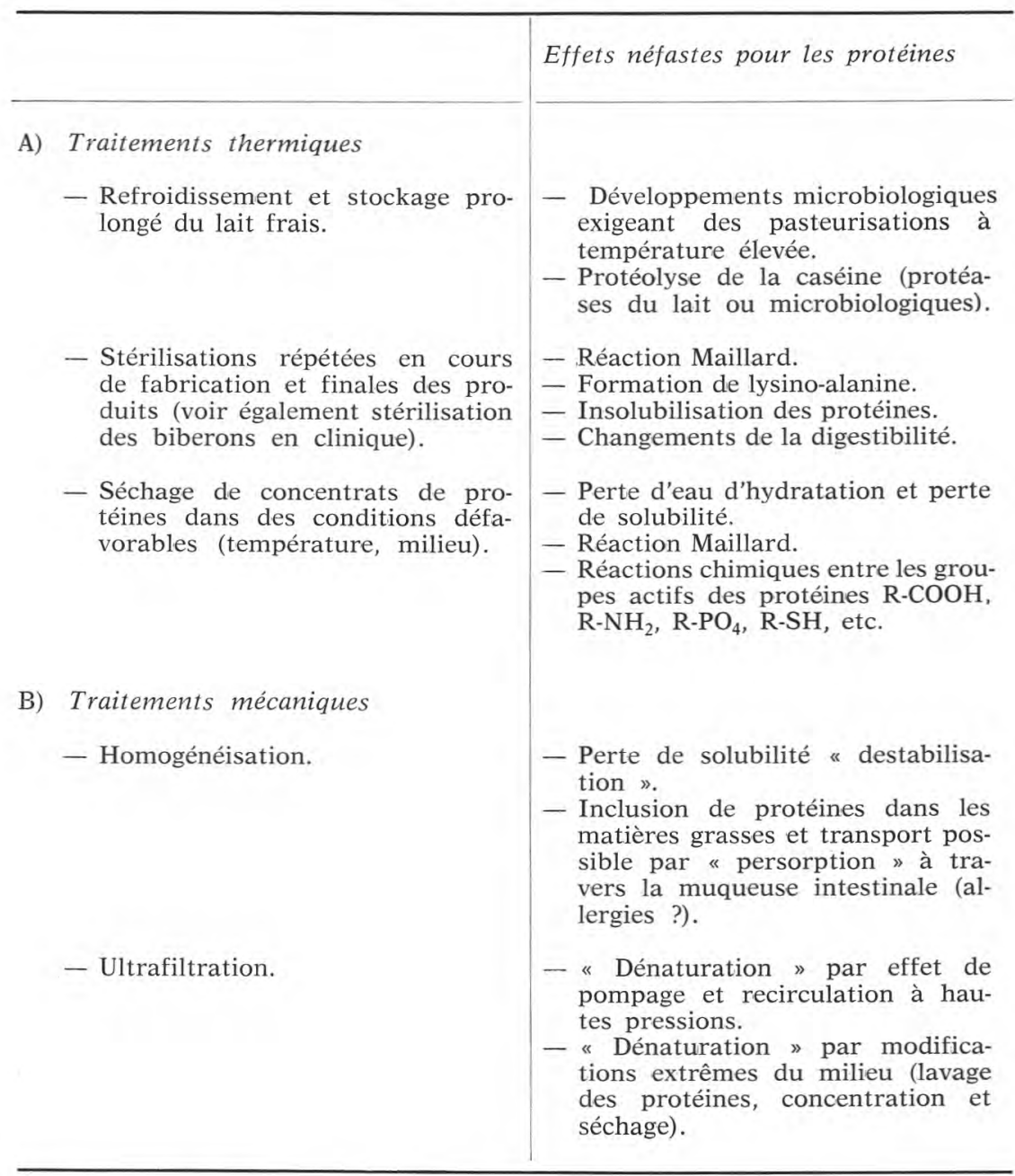

Les technologies disponibles pour extraire les protéines du lait permettent de commencer des études approfondies sur les bienfaits de ces protéines. Ces études pourront prendre modèle sur celles faites sur les animaux, mais elles devront être poursuivies essentiellement sur l'homme vu la complexité des phénomènes apparaissant en nutrition humaine. 
Les immunoglobulines du lait de vache sont actuellement les seules protéines du lait exploitables à des fins thérapeutiques.

La valeur diététique des protéines du lait dépend et dépendra probablement toujours des systèmes alimentaires constitués de plusieurs composés qui devront agir en synergisme.

\section{Bibliographie}

AeBI (H.) (1965). - Einführung in die praktische Biochemie. Akad. Verlags GmbH, Frankfurt.

Braun (O. H.) (1976). - Ueber die infektionsverhütende Wirkung der Mittermilch und deren mögliche Ursachen. Klin. Paediatr., 188, 297.

Braunttzer (G. et al.) (1973). - The sequence of $\beta$-lactoglobulin. Hoppe-Seyter Z. Physiol. Chem., 354, 867.

BREW (K. et al.) (1970), - The complete amino acid sequence of bovine $\alpha$-lactalbumin. J. Biol. Chem., 245, 4570 ,

Brezinowa (V.) and Oswald (I.) (1972). - Sleep after a bedtime beverage. Brit. Med. J., 2, 431.

Bürgin-WolfF (A.) (1980). - The diagnostic significance of antibodies to various cow's milk proteins (fluorescent immunosorbent test). Eur. J. Pediatr., 133, 17.

EHRLich (P.) (1892). - Ueber Immunität durch Vererbung und Säugung. Z. Hyg. Infektions Krankh., 12, 183.

Fernstrom (J. D. et al.) (1974). - Nutritional control of the synthesis of 5-hydroxytryptamine in the brain : in "Aromatic amino-acids in the brain ". Associated Scientific Publishers, P.O. Box 211, Amsterdam.

HALPERN (S. et al.) (1973). - Development of childhood allergy in infants fed breast, soy or cow milk. J. Allerg. Clin. Immunol., 51, 139.

Hambreus (L.) (1978). - Nutritive value of milk protein. $\mathrm{XX}^{\bullet}$ Congrès International de Laiterie, no. 19 ST, Paris.

HILPERT (H. et al.) (1975), - Bovine milk immunoglobulins (Ig), their possible utilisation in industrially prepared infant's milk fomulae. Swedish Nutrition Foundation Symposium no. XIII on Food Immunology, Stockholm.

IIK-NAN CHOU et al. (1979). - Isolation from lactalbumin hydrolysate of a high molecular weight mutagenic factor. J. Biol. Chem., 21, 10588.

Jost (R.) (1982). - Emulgateurs peptidiques obtenus par hydrolyse enzymatique de la protéine du lait. Symposium «Un nouvel avenir pour les protéines du lait », Nancy.

KJellman (N. I. M.) and Johansson (S. G. O.) (1979). - Soy versus cow's milk in infants with biparental history of atopic disease: development of atopic disease and immunoglobulins from birth to 4 years of age. Clinical Allergy, 9, 347.

Klagsbrun (M.) (1978). - Human milk stimulates DNA synthesis and cellular proliferation in cultured fibroblasts. Proc. Nat. Acad. Sci. U.S.A., 75, 5057.

McLaughlan (P. et al.) (1981), - Effect of heat on the anaphylatic-sensitizing capacity of cow's milk, goat's milk and various infant formulae fed to guineapigs. Arch. Dis. Childhood, 56, 165.

Mietens (C. et al.) (1979). - Treatment of infantil E. coli gastro-enteritis with specific bovine anti-E. coli milk immunoglobulins. Eur. J. Pediatr., 132, 239. 
MoLLER (S. E.) (1980). - Relationship between plasma ratio of tryptophan to competing amino-acids and sleeping and eating habits in the healthy females. Biochemical and medical aspects of tryptophan metabolism : proceeding of the Third International Meeting of the international study group for tryptophan research held in Kyoto, Japan, Aug. 4-7, 1980. Editors: O. Hayaiski, Y. Ishimura, R. Kido. Elsevier, North Holland Biomedical Press, Amsterdam.

PAHUD (J. J. et al.) (1981). - Bovine milk antibodies in the treatment of enteric infections and their ability to eliminate virulence factors from pathogenic E. coli. The ruminant immune system. J.E. Butler, Plenum Publishing Corporation.

Petersen (W. E.) and Campbel (B. J.) (1955). - Use of protective principles in milk and colostrum in prevention of disease in man and animals. Lancet, 75, 494.

Ribadeau-Dumas (B.) (1978). - Progrès récents dans la biochimie des protéines du lait. $X^{\circ}$ Congrès International de Laiterie, $n^{\circ} 73$ ST, Paris.

Société des Produits Nestlé S.A. (1967). - Composition cosmétique, hydrolysat enzymatique de lactalbumine. Brevet français $\mathrm{n}^{\circ} 1474747$.

Southwell (P. R. et al.) (1972). - Effect of a hot milk drink on movements during sleep. Brit. Med. J., 2, 429.

WENNER (V.) (1978). - Matières premières lactiques et modifications des produits laitiers traditionnels pour l'orientation de produits diététique. $\mathrm{XX}^{\circ}$ Congrès International de Laiterie, $\mathrm{n}^{\circ} 46 \mathrm{ST}$, Paris.

Wurtmann (R.J.) (1982). - Nutrients that modify brain function. Scientific American, 246, no. 4, 42 . 